\title{
Bariatric surgery:
}

\section{implications for primary care}

\section{BACKGROUND}

Obesity rates in the UK are high with over one-quarter of adults being classified as obese. ${ }^{1}$ Obesity is associated with a number of medical comorbidities, including type 2 diabetes mellitus, hypertension, obstructive sleep apnoea, and osteoarthritis. Consequently, obesity is linked with increased cardiovascular morbidity and mortality, cancer, functional impairment, and premature death, placing a financial burden on the NHS.

Bariatric surgery has been demonstrated to be the most effective method for substantial and sustained weight loss, with a significant reduction in obesity-related comorbidities and long-term mortality. ${ }^{2}$ As a result, bariatric surgery rates are increasing in the UK for patients who have failed to respond to non-surgical therapies. GPs will therefore see an increasing number of patients with severe obesity who wish to be referred for surgery.

\section{ROLE OF THE GP}

GPs play a pivotal role in the management of obesity with early referral of motivated individuals who have failed to achieve substantial weight loss. Further, given the high level of psychosocial disorders in this patient group it is important to make an early assessment and provide support if appropriate. The National Confidential Enquiry into Patient Outcome and Death (NCEPOD) reported that only $29 \%$ of patients received psychological input, which mostly occurred following referral.

Indications for bariatric surgery have been clearly defined by National Institute for Health and Care Excellence (NICE). ${ }^{4}$ However, in light of new evidence NICE has recently issued draft guidance on the management of obesity. ${ }^{5}$ As bariatric surgery has been demonstrated to improve glycaemic control and even remission of type 2 diabetes, NICE recommends that obese patients (body mass index [BMI] 30-34.9) with recent-onset type 2 diabetes (within the past 10 years) may benefit from surgery. Furthermore, patients of Asian origin with recent-onset type 2 diabetes may benefit from bariatric surgery at a lower $\mathrm{BMI}(<30)$. If the updated recommendations are implemented a significant number of individuals may be eligible for bariatric surgery. Limitation of resources, however,

"Bariatric surgery has been demonstrated to be the most effective method for substantial and sustained weight loss..."

will mean that some commissioning groups will have strict criteria, including higher BMI thresholds and thus some patients will not have access to NHS-funded surgery. The National Bariatric Surgery Registry (NBSR) demonstrated that $30.9 \%$ of patients were independently funded. ${ }^{6}$ Prior to referral for surgery, patients need to be made aware of the risks and benefits, including the requirement for lifelong follow-up.

\section{SURGICAL PROCEDURES}

The laparoscopic adjustable gastric band (LAGB) is a restrictive procedure, which artificially creates a small pouch with a narrow outlet. The sleeve gastrectomy (SG) is a restrictive procedure, whereby the stomach volume is reduced in size. The Roux-en-Y gastric bypass (RYGB) is both a restrictive and malabsorptive procedure. The RYGB makes up $>50 \%$ of all bariatric procedures and is associated with the greatest excess weight loss. An appreciation of which procedure patients undergo is important as nutritional deficiencies and physiological problems are more likely when there is a malabsorptive component. The duodenal switch and bilio-pancreatic diversion, both of which have a significant malabsorptive component, are performed rarely.

Once a referral has been made to bariatric multidisciplinary teams, it is usually a while before patients undergo surgery. During this time it is important that dietetic support continues from the GP, as any deviation, such as weight gain, can result in surgery being denied or temporarily withdrawn.

\section{AFTER SURGERY}

Bariatric units should provide clear guidance on the diet to be followed after discharge, including the requirement for multivitamins and lifestyle modification. It is recommended that bariatric surgeons should follow-up patients for 2 years after surgery ${ }^{5}$ and this will be structured, systematic, and team based. Thereafter, the transfer of care will be to primary care although a 'shared care policy will be adopted. However, a number of patients will have their procedure in the private sector or abroad and may not receive regular follow-up. GPs may need to provide the additional support these patients require in close liaison with local bariatric units.

The short-term complications of bariatric surgery include wound infections, vomiting, intolerance to puréed meals, dysphagia (misplaced LAGB), and an anastomotic/ staple line leak (SG/RYGB). Clinical signs, in this early stage, may be subtle and the GP should have a low threshold to urgently refer back to their local bariatric unit.

\section{LONG-TERM PROBLEMS}

In the long term, bariatric patients can develop a plethora of problems, which may require attention from their GP. Dehydration can occur frequently due to poor fluid intake and malabsorption. Dumping syndrome, associated with RYGB, can present with a constellation of symptoms, including abdominal pain, nausea, vomiting, diarrhoea, tachycardia, and syncope. These symptoms, although self-limiting, serve to discourage the intake of energy-rich foods and liquids, and are usually controlled with dietetic advice. Chronic, frequent vomiting may suggest obstruction (such as, anastomotic stricture or internal herniation), reflux, or gastric dysmotility. An internal hernia following a laparoscopic RYGB is potentially fatal and patients may develop symptoms of small bowel obstruction, which necessitate urgent surgical review. Vomiting and overrestriction from an LAGB may require band adjustments or repositioning. Marginal ulceration following an RYGB, although rare, can present with life-threatening haematemesis or melaena. Patients should take regular acid-suppression therapy and avoid non-steroidal anti-inflammatory drugs (NSAIDs). Profound weight loss may be associated with the development of cholelithiasis and, if symptomatic, patients may require a cholecystectomy. Routine cholecystectomy at the time of bariatric 
surgery is rarely performed. Hair loss is observed frequently and can be distressing to the patient, but they should be reassured that it is usually self-limiting.

\section{MALABSORPTION, VITAMIN, AND NUTRITIONAL DEFICIENCIES}

All bariatric procedures lead to a reduced calorie intake $(<1000 \mathrm{kcal} /$ day), with the RYGB carrying the greatest risk of nutritional deficiencies. By 2 years most patients have established a balanced diet; however, issues may still arise. Proteincalorie malnutrition (albumin $<35 \mathrm{~g} / \mathrm{L}$ ), the most severe macronutrient complication, can be avoided by ingesting a diet rich in protein ( $\geq 60 \mathrm{~g} /$ day), with carbohydrates and fat added as determined by energy needs. However, micronutrient deficiencies are more common after surgery. Iron deficiency is the most common, particularly in menstruating women and as such patients require daily iron supplementation. Vitamin B12 deficiency is common, particularly in the long term, when reserves become depleted. Patients require B12 supplementation (intramuscular injection every 3-6 months) above and beyond a multivitamin. Patients can also have deficiencies in thiamine, folate, and vitamin B6. Hypovitaminosis D land hypocalcaemial is common in obese patients, even prior to surgery and is related to a sedentary lifestyle and reduced exposure to sunlight. Consequently, secondary hyperparathyroidism with bone demineralisation may occur following surgery. Thus, lifestyle modification as well as supplementation (Calcichew D ${ }^{\circledR}$, Shire Pharmaceuticals) is necessary following surgery. Steatorrhoea, secondary to malabsorption, may result in deficiencies in fat-soluble vitamins (A, D, E, and $K)$.

Determining the risk of developing micronutrient deficiencies is difficult, as no consensus exists on the amount of vitamin and mineral supplementation required. Furthermore, deficiencies cannot be prevented with supplementation alone. ${ }^{\text {? }}$ GPs play a key role in ensuring compliance with multivitamin supplementation, which is often neglected by patients years after surgery. Annual monitoring is recommended ${ }^{5}$ and should at a minimum include a full blood count, electrolytes, liver function tests, and serum levels of glucose, iron, ferritin, vitamins D, B12, calcium, parathyroid hormone, thiamine, folate, and selenium.

\section{CHRONIC DISEASES FOLLOWING BARIATRIC SURGERY}

Bariatric surgery has been shown to result in remission of a number of obesity-related comorbidities. ${ }^{8}$ Remission of type 2 diabetes mellitus has been demonstrated, with insulin and oral hypoglycaemics likely to be reduced in dose or no longer required. ${ }^{9}$ The long-term management following remission of type 2 diabetes mellitus is not established but during follow-up a $\mathrm{HbA1c}$ level should be performed. Effects on hypertension, hyperlipidaemia, and obstructive sleep apnoea ${ }^{8}$ are also evident, and patients should be periodically evaluated.

\section{PREGNANCY FOLLOWING BARIATRIC SURGERY}

After surgery female patients may discover that they are now able to conceive, something they previously failed to achieve. However, this is not recommended for at least 12-18 months after surgery because of rapid weight loss and nutritional deficiencies. Indeed, perinatal outcomes following bariatric surgery include an increased risk of preterm and small-forage births. ${ }^{10}$ Therefore, GPs need to develop an appropriate contraceptive strategy. For those patients who do conceive, referral to an obstetric unit with access to a bariatric unit is advised. Deficiencies need to be identified and additional vitamin and calcium supplementation are often necessary.

\section{WEIGHT LOSS AND WEIGHT REGAIN}

Patients need a realistic expectation of excess weight loss and that the goal is not an 'ideal weight', but a 'healthy weight' that will reduce long-term issues. Excess weight loss at 1 year can be as high as $57.8 \%$, with patients plateauing after 2 years. GPs play a crucial role, ensuring compliance with dietary and lifestyle recommendations. Weight regain is not uncommon and is predominantly related to noncompliance, although surgical failure can be a cause and thus patients need re-referral to a bariatric surgeon.

\section{CONCLUSION}

GPs may be the only medical professionals involved years after surgery is performed and it is essential that long-term surveillance is continued. Importantly, primary care requires the infrastructure to provide adequate support for this complex group of patients.

\section{Vamshi P Jagadesham,}

Senior Registrar in Upper GI Surgery, Northern General Hospital, Sheffield.

\section{Marion Sloan,}

GP, Sloan Medical Centre, Sheffield.

\section{ADDRESS FOR CORRESPONDENCE}

Roger Ackroyd

Northern General Hospital, Herries Road, Sheffield, South Yorkshire, S5 7AU, UK.

\section{E-mail: roger.ackroydasth.nhs.uk}

\section{Roger Ackroyd,}

Consultant in Upper GI \& Bariatric Surgery, Northern General Hospital, Sheffield.

\section{Provenance}

Commissioned; not externally peer reviewed.

DOI: 10.3399/bjgp14X680797

\section{REFERENCES}

1. Health \& Social Care Information Centre. Statistics on obesity, physical activity and diet - England, 2014. http://www.hscic.gov. uk/catalogue/PUB13648/Obes-phys-acti-dieteng-2014-rep.pdf (accessed 14 Jul 2014).

2. Sjöström L, Narbro K, Sjöström D, et al. Effects of bariatric surgery on mortality in Swedish obese subjects. N Engl J Med 2007; 357(8): 741-752.

3. National Confidential Enquiry into Patient Outcome and Death. Bariatric surgery: too lean a service? NCEPOD, 2012. http://www. ncepod.org.uk/2012bs.htm laccessed 7 Jul 2014).

4. National Institute for Health and Care Excellence. Obesity: guidance on the prevention, identification, assessment and management of overweight and obesity in adults and children. London: NICE, 2006. http://guidance.nice.org.uk/CG43 laccessed 7 Jul 2014).

5. National Institute for Health and Care Excellence. NICE consults on updated recommendations for treating obesity. http:// www.nice.org.uk/nice-consults-on-updatedrecommendations-for-treating-obesity (accessed 14 Jul 2014).

6. Welbourn R, Fiennes A, Kinsman R, Walton P. National bariatric surgery registry: first registry to March 2010. Henley-on-Thames: Dendrite Clinical Systems Ltd, 2011.

7. Gasteyger C, Suter M, Gaillard RC, Giusti $\checkmark$. Nutritional deficiences after Roux-en-Y gastric bypass for morbid obesity often cannot be prevented by standard multivitamin supplementation. Am J Clin Nutr 2008; 87(5): 1128-1133.

8. Buchwald $\mathrm{H}$, Avidor $Y$, Braunwald $\mathrm{E}$, et al. Bariatric surgery: a systematic review and meta-analysis. JAMA 2004; 292(14): 17241737.

9. Brethauer SA, Aminian A, Romero-Talamas $\mathrm{H}$, et al. Can diabetes be surgically cured? Long-term metabolic effects of bariatric surgery in obese patients with type 2 diabetes mellitus. Ann Surg 2013; 258(4): 628-636.

10. Roos N, Neovius M, Cnattingius $S$, et al. Perinatal outcomes after bariatric surgery: nationwide population based matched cohort study. BMJ 2013; 347: f6460. 